\title{
EchoGéo
}

$14 \mid 2010$

Afrique, 50 ans d'indépendance : Dynamiques spatiales, identités, circulations

\section{L'élaboration des nouveaux programmes du Lycée : une question de compromis}

\author{
Entretien avec François Louveaux, IGEN
}

\section{Alexis Sierra}

\section{(2) OpenEdition}

\section{Journals}

\section{Édition électronique}

URL : https://journals.openedition.org/echogeo/12173

DOI : $10.4000 /$ echogeo. 12173

ISSN : 1963-1197

\section{Éditeur}

Pôle de recherche pour l'organisation et la diffusion de l'information géographique (CNRS UMR 8586)

\section{Référence électronique}

Alexis Sierra, «L'élaboration des nouveaux programmes du Lycée : une question de compromis »,

EchoGéo [En ligne], 14 | 2010, mis en ligne le 16 décembre 2010, consulté le 01 août 2021. URL : http:// journals.openedition.org/echogeo/12173; DOI : https://doi.org/10.4000/echogeo.12173

Ce document a été généré automatiquement le 1 août 2021.

EchoGéo est mis à disposition selon les termes de la licence Creative Commons Attribution - Pas d'Utilisation Commerciale - Pas de Modification 4.0 International (CC BY-NC-ND) 


\section{L'élaboration des nouveaux programmes du Lycée : une question de compromis}

Entretien avec François Louveaux, IGEN

Alexis Sierra

1 François Louveaux est Inspecteur général de l'Education Nationale. Agrégé de géographie et normalien, il a été professeur dans le secondaire puis durant vingt-huit ans en classe préparatoire à Poitiers puis au lycée Henri IV à Paris. Il a été membre des jurys de l'agrégation interne et externe. Il a une longue expérience de la formation continue des enseignants et il est aujourd'hui plus particulièrement en charge des dossiers "post-baccalauréat " et de l'articulation entre enseignement secondaire et supérieur. Il s'exprime ici à titre personnel, ses propos n'engagent pas l'Inspection générale. L'interview a été effectuée dans les locaux d'Echogéo, le 30 septembre 2010.

2 - Alexis Sierra (AS). François Louveaux, bonjour. Avant d'entrer dans le contenu des nouveaux programmes de géographie du lycée, pourriez-vous nous rappeler le contexte dans lequel ils ont été élaborés?

3 - François Louveaux (FL). La réforme des programmes est consécutive à la réforme du lycée. Jusqu'ici l'histoire et la géographie étaient présentes dans toutes les voies et séries du primaire jusqu'en terminale, sauf dans certaines séries technologiques, industrielles notamment, où elles étaient présentes jusqu'en première. Ces disciplines sont devenues optionnelles en terminale scientifique; en première scientifique leur horaire a été porté à 4 heures, comme en L et ES.

4 Cette forte présence de l'histoire - géographie signifie que les programmes doivent être pensés dans leur globalité du primaire jusqu'au supérieur, avec une progression régulière. Il faut tenir compte aussi de l'instauration du «socle commun» (de connaissances et de compétences) qui concerne l'ensemble de la scolarité obligatoire.

5 Le programme de collège doit permettre de couvrir une première fois la totalité du spectre des thèmes et des problématiques puisque la fin du collège marque globalement 
la fin de l'école obligatoire. Il est impossible d'envisager de réserver un certain nombre d'éléments pour le lycée; le programme de collège doit donc constituer un tout. Le programme de lycée est fixé en fonction de ce qui a été fait au collège et de ce qui est supposé être connu ou retenu. Désormais il y a aussi la volonté d'articuler le programme du lycée avec ce qui se fera à l'université. Ce dernier point a été exprimé très clairement. En particulier, la terminale doit être un entraînement à ce que sera l'université. Reste à définir ce qu'est l'université et ce que sont les besoins de l'université...

6 Il faut bien comprendre qu'il y a des contraintes très lourdes, qui sont des contraintes externes...

7 - AS. Justement, la question est de comprendre comment s'élaborent les programmes sachant effectivement qu'il y a à respecter des contraintes et des exigences...

8 - FL. Comprendre qu'il y a des contraintes, c'est aussi comprendre que forcément il y a des compromis.

9 - AS. .....pédagogiques, scientifiques, politiques...

10 - FL. Tout à fait. Tout programme est forcément le résultat de compromis. L'une des tâches de l'Inspection générale au niveau national et des Inspections pédagogiques régionales, c'est de bien mettre au clair les choix qui ont été faits, les pistes qui s'offraient et le pourquoi des choix qui ont été faits. Cela n'entre pas dans le texte du programme et il faut l'exposer lors de sa mise en application.

11 - AS. Concrètement, quel est le circuit ? Qui décide?

12 - FL. Tout part d'une demande ministérielle. C'est le ministère, le ministre et son cabinet, qui en fonction d'évolutions qui n'ont pas à voir directement avec l'histoire géographie (par exemple la mise en place du socle commun ou la réforme du lycée) exprime la demande de transformation des programmes. Le ministre passe commande à la Direction générale de l'enseignement scolaire (DGESCO). Celle-ci mandate un groupe d'experts. Ces groupes d'experts sont généralement co-pilotés par un Inspecteur général et un universitaire. Ces groupes d'experts sont relativement restreints: pour l'histoire - géographie, deux universitaires- un géographe, un historien - deux Inspecteurs généraux - un géographe, un historien - deux Inspecteurs pédagogiques régionaux - un historien, un géographe, et deux enseignants du secondaire en exercice - un géographe, un historien. Le groupe d'experts a tout loisir de consulter des personnes qualifiées et notamment des spécialistes universitaires. Les syndicats et les associations professionnelles comme l'APHG (Association des professeurs d'histoire et de géographie) par exemple, sont également consultés en amont.

13 Ensuite le programme préparé par le groupe d'experts est soumis à consultation. Le ministère le met en ligne sur le site eduscol en vue d'une large consultation des enseignants.

14 - AS. C'est donc une consultation publique?

15 - FL. Oui, publique et nationale. Les enseignants peuvent faire part de leurs remarques. Dans un souci d'efficacité, ce sont souvent les Inspecteurs pédagogiques qui, au niveau régional lancent la consultation, regroupent les participations et en font la synthèse qui est transmise au Ministère. Ensuite le groupe d'experts se réunit à nouveau pour finaliser le programme en tenant compte de ces «remontées de terrain». C'est seulement après la remontée, après les modifications éventuelles que le texte du 
programme devient définitif. Ce n'est pas un leurre parce que, je crois que beaucoup de collègues ont été surpris par l'ampleur des remaniements du programme de seconde.

18 Le programme est ensuite soumis pour avis au Conseil supérieur de l'éducation (CSE). Le CSE est un organisme paritaire qui regroupe des représentants de l'administration, dont des Inspecteurs généraux, des représentants de la DGESCO, des représentants des fédérations de parents d'élèves, des représentants des enseignants par l'intermédiaire des syndicats, des représentants des lycéens par l'intermédiaire des associations lycéennes et quelques personnalités extérieures. Le CSE a un avis consultatif. Chose extraordinaire soulignée par le ministre, les programmes d'histoire - géographie de seconde et de première ont été validés par le CSE. Ce qui est, paraît-il, relativement rare.

19 Ensuite le programme est publié par le ministre et s'impose. Commence alors toute une phase, essentielle, de communication, d'aide à la mise en oeuvre du programme, qui passe notamment par l'organisation de regroupements inter académiques organisés par l'Inspection générale et par la réalisation de " ressources pour faire la classe », élaborées sous la responsabilité de l'Inspection générale et mises en ligne sur eduscol. Il s'agit de fiches de 2/4 pages sur chacune des questions pour essayer de cadrer les attentes et d'expliquer les choix qui ont été faits, ce vers quoi il faut aller, pour essayer de guider les enseignants. Des réunions académiques sont également organisées par les Inspecteurs pédagogiques régionaux qui ont mis en place des groupes de formateurs pour aider à cette mise en œuvre.

20 - AS. Comment sont choisis les membres du groupe d'experts?

21 - FL. Cela relève du Directeur Général de l'Enseignement Scolaire. Il demande au doyen de l'Inspection générale de proposer un Inspecteur Général pour présider le groupe d'experts. Ce dernier, en accord avec le DEGESCO, arrête ensuite la composition du groupe.

22 - AS. Or, tout ce parcours d'élaboration du programme s'est fait cette fois-ci dans un laps de temps réduit, de façon précipitée.

23 - FL. Précipité non, ce fut rapide !

24 - AS. C'est le terme!

25 - FL. Il faut bien voir que l'impulsion est politique. C'est le ministre qui, en fonction de la réforme du lycée, a demandé aux différents groupes de l'Inspection générale et aux différents groupes d'experts de prévoir une adaptation, une évolution des programmes. Comme le calendrier de mise en place de la réforme était extrêmement réduit, c'est vrai que les groupes d'experts ont eu cette année peu de temps pour travailler. Le calendrier était contraint. En revanche, pour la classe de première, le calendrier le fut un peu moins, et il y a presque un an pour le programme de terminale puisque la commande est pour février 2011, date de la consultation. Cela laisse un peu plus de temps pour réfléchir au programme de terminale qui est d'autant plus complexe que la grande nouveauté c'est la disparition de l'obligation de l'histoire - géographie en classe de terminale scientifique. Cela va obliger à penser un examen en fin de première pour 
les élèves de cette série et un autre en fin de terminale pour les élèves des séries L et ES qui sera différent après une année supplémentaire de formation. Cela a conduit aussi à faire des choix délicats pour les programmes de seconde et de première.

Il a fallu réduire un programme qui, depuis des lustres, était conçu sur 3 ans et savoir quels contenus étaient indispensables pour un élève qui ne continuerait pas ensuite l'histoire - géographie en terminale scientifique.

27 - AS. Pourtant la classe de seconde est a priori indéterminée ?

28 - FL. Oui. En première, il y a un début de commencement de choix pour la suite mais la réforme du lycée c'est bien l'idée que le choix d'orientation se fait en terminale et qu'on espère un choix beaucoup plus large qu'actuellement où finalement une section, la section $S$, regroupe les meilleurs élèves quelle que soit leur tonalité.

29 - AS. Et qu'est-ce qui est prévu en terminale?

30 - FL. Il y aura deux programmes pour la terminale: le programme des L/ES et le programme de l'option proposée aux scientifiques. Il faut donc réfléchir à une nouvelle épreuve écrite en fin de terminale pour les L et ES et à une épreuve de validation en fin de terminale pour les élèves du bac scientifique qui auront pris l'option. Ce serait a priori une épreuve orale, mais sous quelle forme? On peut imaginer par exemple que ce serait la production d'un dossier. Les choix qui vont être faits sont des choix stratégiques pour l'histoire et la géographie.

31 - A.S. Surtout si, comme vous disiez tout à l'heure, il faut intégrer la perspective de l'université.

32 - FL. Oui, l'enjeu est de taille car près de $40 \%$ des géographes aujourd'hui à l'université ont fait une Terminale scientifique.

33 - AS. La géographie en Terminale était cependant déjà différenciée entre $\mathrm{S}$ et les autres filières générales.

34 - FL. Oui, il y avait un allègement du programme des scientifiques puisqu'ils avaient $2 \mathrm{~h} 30$ de cours d'histoire géographie par semaine, tandis que les élèves de L et ES bénéficiaient de 4 heures.

35 - AS. Sauf que cette fois-ci les élèves de la filière scientifique s'arrêtent à la fin de la première. Comment gérer cette absence de formation pour des élèves qui voudraient faire des études de géographie par la suite et comment gérer le déroulement de la formation pour l'ensemble des filières?

36 - FL. Pour les scientifiques, la Première, c'est le programme de fin d'étude obligatoire. Sauf que nous espérons qu'ils seront massivement attirés par l'option HistoireGéographie en Terminale et que cette option sera en effet mise en place dans tous les lycées. En revanche, pour les élèves de L/ES ce n'est qu'une étape: il n'y a pas d'examen. Il faut donc arriver à trouver un programme qui permette les deux orientations. C'est un franc changement parce qu'actuellement le programme de Première laisse une certaine marge de manœuvre dans la mesure où il n'y a pas d'examen en fin d'année. Les collègues peuvent se donner plus de liberté. Désormais, en revanche, la responsabilité c'est aussi de préparer l'examen.

37 - AS. Y aura-t-il une obligation de l'offre d'option pour les Terminale S ?

38 - FL. Il semble en effet essentiel qu'elle puisse être partout présente mais c'est une décision ministérielle. 
39 - AS. Avez-vous envisagé le risque de dévalorisation de la géographie (et de l'histoire) dans cette suppression de l'obligation?

40 - FL. Deux éléments de réponse. C'est une décision ministérielle qu'il nous appartient donc de mettre en œuvre. L'important c'est désormais de réussir dans cette nouvelle configuration: proposer aux $\mathrm{S}$ un enseignement attractif, une épreuve valorisante, montrer tout l'intérêt de cet enseignement d'Histoire-Géographie pour un futur scientifique ou un candidat à des écoles de management, par exemple. Proposer aux L et ES un enseignement rénové, actuel, qui prépare à l'enseignement supérieur, mais puisse aussi faire l'objet d'une épreuve intéressante, réaliste, valorisante au Bac. L'équation n'est pas simple, mais le défi mérite d'être relevé.

41 Un autre élément doit entrer dans ce schéma : la réforme du lycée prévoit qu'à côté des enseignements obligatoires, il y a des enseignements dits "d'exploration». Or, les historiens et les géographes ont demandé, et obtenu, que leur discipline soit présente dans certains de ces enseignements d'exploration. Il faut encore là aussi imaginer d'autres types d'approches, d'autres types de supports. En Seconde, l'enseignement d'exploration « Littérature et Société » doit être assuré conjointement par le professeur de français et le professeur d'Histoire-Géographie. En Terminale, il y aura un enseignement optionnel lourd, de 3 ou 4 heures sur «Droits et enjeux du monde contemporain ».

42 - AS. Quelle est l'orientation de cet enseignement?

43 - FL. Il doit être conçu en articulation avec l'ECJS [Education civique, juridique et sociale]. Les historiens géographes seront représentés dans le groupe d'experts, comme ils le sont dans le groupe ECJS.

44 - AS. Dans l'élaboration des programmes, outre ces contraintes institutionnelles, il y a des contraintes pédagogiques. Or, certaines notions à enseigner sont complexes, comme celle de développement durable. L'interrogation porte sur le choix même de cette notion: pourquoi avoir fait du développement durable, le fil directeur des programmes?

45 - FL. Les programmes d'Histoire comme de Géographie sont marqués par des héritages extrêmement lourds. Quand on regarde les programmes sur plus d'un siècle, on voit une très grande filiation : la France, l'Europe en première puis le monde en Terminale. Par ailleurs, il y a la géographie générale, surtout en seconde. Les programmes de géographie ont cependant beaucoup évolué dans le contenu depuis quelques décennies et singulièrement durant la dernière. La géographie universitaire s'est en particulier défaite de la tripartition classique géographie physique, géographie humaine, géographie régionale pour d'autres types d'approches. Très rapidement, c'est un cas suffisamment rare pour le souligner, la géographie scolaire a suivi cette évolution et s'est affirmée comme une géographie des sociétés. Cela a eu des échos sur la façon d'envisager l'histoire, puisque le cours d'histoire géographie a clairement pour objet des sociétés dans leur histoire et dans leurs espaces, dans leurs territoires. La géographie scolaire a pris extrêmement vite ce tournant-là au dam d'un certain nombre de collègues qui trouvaient beaucoup d'attraits à la géographie traditionnelle, physique en particulier : elle est toujours présente mais reprise désormais sous l'angle des risques et de l'environnement. La géographie a évolué très vite dans ses approches avec un angle social extrêmement clair. De la même façon, elle a pris en compte très 
rapidement un certain nombre de concepts, comme le territoire, le risque, de démarches comme l'emboîtement des échelles.

Dans ses pratiques l'enseignement de la géographie a mis en avant ce qu'on a appelé l'étude de cas, donc une démarche inductive en se disant que finalement à partir d'un exemple précis, on peut montrer l'exceptionnelle complexité d'un phénomène et la multitude d'acteurs. C'est un choix qui s'inscrit dans les programmes depuis une dizaine d'années. En histoire l'étude précise d'un monument, d'un personnage, d'une corporation, d'un fait historique, peut-être un élément pour la compréhension de phénomènes plus larges.

47 - AS. Le bouleversement a été plus fort dans l'enseignement de la géographie.

48 - FL. Il a été plus fort et extrêmement rapide à l'échelle du temps. Il se mesure aussi dans les thèmes mis aux concours. Dans le cas de l'évolution de la discipline et de son enseignement, le pilotage des concours de recrutement du CAPES et de l'Agrégation est un élément essentiel. C'est un des moyens pour faire passer des thématiques et des méthodes de travail. A propos de la nouvelle mouture du CAPES et de l'articulation CAPES/Agrégation il y a eu assez peu de débats en géographie, les jurys de CAPES et de l'Agrégation étant à peu près sur la même longueur d'ondes.

49 L'objectif des examens est aussi un facteur d'évolution de la géographie scolaire. L'épreuve d'examen détermine en partie ce que l'on peut mettre dans les programmes. Il faut identifier ce qui est réaliste parce qu'au bout du compte, le but est aussi que les élèves qui ont travaillé aient une bonne note. Ceux qui sont brillantissimes, tant mieux pour eux, auront une très bonne note mais on n'est pas là pour piéger les candidats. Il y doit donc y avoir un vrai souci de faisabilité dans l'élaboration des programmes.

50 - AS. Comment ces évolutions de la géographie scolaire expliquent-elles pour partie, l'introduction de la notion de développement durable?

51 - FL. La géographie s'affirme comme sociale. La géographie se veut un outil pour comprendre les enjeux du monde contemporain. La géographie ne peut donc pas se désintéresser des questions qui agitent la société contemporaine.

52 - AS. Sinon elle retombe en crise comme dans les années 70.

53 - FL. Il y a une finalité intellectuelle, il y a une finalité culturelle et puis il y a une finalité civique. Il faut aider des adolescents à devenir des citoyens actifs, éclairés, leur permettre de se construire, leur donner des éléments de connaissance, de compréhension du monde. Quand on prétend être une science de la société, du moins en prise avec la société actuelle, c'est tout à fait logique de traiter de la mondialisation ou du développement durable. La difficulté est de savoir ce qu'on met derrière les mots. On n'y met sans doute pas la même chose au collège qu'au lycée parce qu'on n'a pas affaire aux mêmes élèves. L'idée essentielle c'est de bien faire comprendre que la mondialisation et le développement durable ne sont conçus que comme des grilles de lecture possible du monde. Dans l'exceptionnelle diversité des phénomènes qui font le monde actuel, deux éléments sont particulièrement significatifs des débats, des réalités et des enjeux. Je mets en parallèle mondialisation et développement durable parce que c'est le même type de réflexe qu'on doit avoir face à ces objets. Le but n'est pas de dire vive l'altermondialisme ou pas : ce sera le libre choix des élèves de s'engager ou non dans une ONG, par exemple. Nous, nous sommes là pour leur donner des outils qui, après, leur permettront de faire leur choix de façon éclairée. Il ne s'agit pas de transformer le développement durable en une sorte de catéchisme. Je crois que l'on 
confond assez régulièrement l'étude du développement durable avec l'éducation au développement durable, une sensibilisation au respect de l'environnement, aux économies d'énergie entre autres, qui est une injonction citoyenne. Le développement durable n'est jamais qu'une grille de lecture possible qui a des avantages, des inconvénients et ses présupposés. Le programme de géographie de seconde ne s'intitule pas « développement durable », mais « sociétés et développement durable ».

54 - AS. L'équilibre qui définit le développement durable, entre question écologique, sociale et économique, l'équilibre entre les 3, est différent selon l'interlocuteur, selon celui qui l'utilise.

55 - FL. Ce qui est intéressant, même avec des élèves de collège (le développement durable apparaît comme le thème clé des programmes dès la $5 \mathrm{e}$, puis en suite en seconde générale et en seconde bac pro) c'est de faire prendre conscience qu'une action sociale ou une action économique ont des conséquences sur l'environnement, que la préservation de l'environnement a des implications sociales et économiques, qu'il y a des interrelations entre les trois. L'idée est de sortir d'une vision de l'environnement qui serait uniquement une vision naturaliste : ça c'est notre métier !

56 - AS. Le développement durable pose beaucoup de problèmes aux géographes et je me demandais pourquoi avoir utilisé le terme de développement durable et pas celui d'environnement qui était déjà dans le programme de Seconde. Un chapitre obligatoire était ainsi intitulé "Dynamiques urbaines et environnement urbain». Dans les nouveaux programmes, le titre est devenu ville et développement durable. Pourquoi ne pas continuer à utiliser le terme d'environnement qui finalement mêle étroitement société et nature?

57 - FL. Encore une fois, je crois que le souci était de montrer que l'enseignement d'histoire-géographie est ancré dans la réalité sociale contemporaine et qu'il a quelque chose à dire sur ces réalités-là. Ensuite, il y a un effet d'affichage que quelques-uns considèrent comme un peu lourd avec développement durable partout. Il y a pourtant eu allégement entre la version de départ et la version remontée de consultation...

58 L'intérêt du développement durable, c'est de montrer qu'il y a des interactions entre 3 piliers qu'on ne peut pas dissocier les uns des autres. Faire de la systémique, c'est tout de même cela que l'on veut faire en géographie. Surtout au lycée, on est là pour montrer au contraire les interrogations, les contradictions. Je crois que le développement durable, c'est aussi un enseignement des contradictions.

59 - AS. Il y a cependant une difficulté pédagogique parce qu'on parle déjà de développement durable dès la $5 \mathrm{e}$, à un niveau où l'on tend à donner une connaissance intangible et moins des notions qui sont relatives et complexes. J'imagine que sur les villes durables par exemple, on peut dire "les villes durables c'est la ville recyclée, compacte, démocratique, ... ", une sorte de prêt à penser qui vient des institutions internationales, mais qui a l'avantage d'être facile parce que ça donne des repères fixes.

60 - FL. Oui, ce serait catastrophique. Ce n'est pas du tout notre volonté. L'esprit critique est essentiel dans la formation. On est là pour justement dépasser les discours convenus. Ce n'est pas un appel à l'endoctrinement mais au contraire à la raison critique. Au collège, on ne peut guère aller au-delà de la mise en garde, de l'appel à la vigilance critique. Au lycée on peut « démonter » le développement durable : il apparaît à un moment, dans certains cercles, son succès correspond aussi à une certaine sensibilité sociale, il connaît un certain nombre d'évolutions. Tout cela il faut le dire. 
61 Pour la notion de mondialisation, c'est pareil. Le danger, c'est de donner l'impression qu'il y a une espèce de deus ex machina, de phénomène mécanique, qui ne laisse comme alternative que de résister ou de suivre, parce ce qu'on ne peut pas faire autrement. Notre métier, c'est d'expliquer des phénomènes imbriqués, qui montrent l'évolution du monde actuel, qu'on a regroupés et désignés sous ce terme. C'est la difficulté des programmes de conjuguer l'enseignement de la complexité et du plus contemporain tout en voulant être simple et compréhensible, le pari est extrêmement complexe, mais stimulant.

62 - FL. La mondialisation s'est imposée extrêmement vite puisqu'il y a des géographes qui ont travaillé dessus mais les programmes n'ont jamais posé franchement la question de la légitimité du terme. En revanche, le développement durable a été dès le départ une notion suspecte aux yeux d'un certain nombre de chercheurs et d'enseignants car assimilée à une sorte de morale.

63 - AS L'idée avec la notion de développement durable, c'est certes de sensibiliser sur des questions environnementales avec des problèmes de ressources, de pollution, des risques. Mais il y a le terme de développement : on y retrouve en fait une géographie du développement qui n'apparaissait plus en tant que telle si ce n'est en distinguant toujours des Nords et des Suds par exemple, ou différents niveaux de développement à l'intérieur d'un pays.

64 - FL. Oui tout à fait. Dans l'esprit des concepteurs des programmes c'est bien de réintroduire le développement. C'est une autre façon de penser la notion de développement qui est une notion extrêmement complexe et discutée : quand on regarde de très anciens programmes où il y avait la Côte d'Ivoire présentée comme un modèle et une voie de développement, cela apparaît aujourd'hui comme une cruelle et totale désillusion; c'est un peu le cas de l'étude des réformes agraires en Amérique Latine : à un moment donné ce fut pertinent, là ça ne l'est plus. Il est intéressant de montrer comment des éléments apparaissent et disparaissent, pourquoi ils apparaissent et pourquoi ils disparaissent. C'est là vraiment dans notre rôle de formateur. Un certain nombre d'exemples d'aménagement durable montrent qu'il peut être aussi un outil d'exclusion sociale. Pour certains auteurs, avec le développement durable, on refait aux Sud ce qui avait été fait avec le développement : n'est-ce pas aussi un moyen de domination?

65 - AS. Cela implique une formation aussi des enseignants parce que c'est une question complexe et récente. Quelles sont les orientations qui sont prévues pour accompagner ces programmes?

66 - FL. C'est un problème fondamental. Il faut un moment pour maîtriser les programmes. Or, une fois qu'un professeur est parti sur une voie, le cours est fait; il a trouvé ses petites accroches, ses exercices. Il faut donc faire vite pour lui indiquer s'il a pris une mauvaise voie et l'aider à faire autrement.

67 - AS. C'est pourquoi nous sommes à un moment clé.

68 - FL. C'est le rôle de l'Inspection d'être un conseil. L'enjeu de la formation [continue] est un enjeu majeur. C'est ce qui donne la cohérence d'ensemble. Il y a certes eu des débats, des publications, des colloques entre spécialistes sur des notions comme celles-ci et c'est essentiel. Cependant, je crois profondément qu'il faut faire des journées de formation sur le même thème en réunissant des universitaires, des formateurs de l'IUFM et des collègues. Les enseignants de lycée et de collège ont besoin d'échanger 
avec des universitaires. Simplement, il faut prendre en compte ce que c'est que l'enseignement au jour le jour. Il faut donc permettre aux enseignants de prendre du recul, leur apporter des lectures et des éclairages qu'ils n'ont pas forcément. La question est donc moins dans les programmes qu'elle n'est dans la formation.

- AS. Le tout est de savoir si effectivement, les moyens de cet accompagnement existent.

70 - FL. S'il y a un message à faire passer, c'est celui-là. La formation scientifique et pédagogique me parait être un élément indispensable pour résoudre ces contradictions. Ce qui est tout à fait étonnant, c'est que celui qui donne le «la», c'est l'auteur des manuels. L'interprétation du programme est faite par la lecture qu'en donnent les manuels. Les programmes changent, amenant des notions, des concepts, des démarches auxquels les enseignants ne sont pas forcément habitués ou même préparés. Les enseignants font beaucoup appel, on le leur a suffisamment dit, aux documents et, par conséquent aux manuels qui en sont la principale source. Ceux-ci proposent des utilisations, des interprétations et jouent donc un rôle considérable. Or, les manuels ont été produits avec d'autres logiques, sans être forcément en parfaite adéquation avec l'esprit du programme. Il y a donc un effort à faire au niveau de la formation continue. Il y a dans l'Institution, les CRDP, les Centres régionaux de documentation pédagogique, le Centre national de documentation pédagogique (CNDP), le Centre national d'enseignement à distance (CNED), l'Institut national de recherche pédagogique (INRP), sont autant d'organismes qui dépendent de la puissance publique mais dont le rôle, l'articulation, la coordination ne sont pas toujours très nets. Les moyens électroniques démultiplient les possibilités. Cependant, les sites seuls ne suffisent pas toujours, il faut du présenciel.

71 - AS. Je voulais rentrer à l'intérieur du programme de Seconde. En dehors de ce fil directeur du développement durable, existe-t-il des éléments de modification qui vous ont paru notables?

72 - FL. En fait, il reprend très largement le programme antérieur. Il y a davantage de choix optionnels parce que là aussi les contraintes sont claires. Les programmes guident et laissent en même temps des espaces de choix. Ça fait partie des compromis. Il faut en particulier que le programme soit réaliste, qu'il puisse être bouclé, puisqu'il a une continuité d'une année à l'autre.

73 - AS. Certains thèmes ont été introduits, l'enjeu énergétique par exemple. « Nourrir les hommes " et "L'eau " étaient déjà présents mais ils étaient obligatoires alors que maintenant ils sont au choix avec l'enjeu énergétique. J'imagine que là aussi c'est pour comprendre le monde actuel, avec les crises qu'il y a pu avoir sur l'énergie...

74 - FL. Tout à fait. Surtout que ce nouveau programme de Seconde, c'est un programme de bac - 1 pour des scientifiques. Il y a des éléments qui faisaient partie du programme de Terminale qu'il fallait déjà introduire ici. La grande différence c'est que les élèves de Terminale scientifique n'auront pas eu ce qu'ils avaient depuis une éternité, c'est-àdire un panorama global du monde contemporain, qui a pris des noms différents, "mondialisation", "grandes puissances", « développement». Pour les S, le programme obligatoire s'arrêtera à la France et l'Europe dans la mondialisation et il faut donc introduire une vision $d u$ monde en Seconde. Cette vision du monde apparaitra également en Terminale scientifique en option. L'idée est donc de proposer en Seconde un petit gâteau un peu appétissant intellectuellement pour que des élèves 
de Terminale scientifiques se disent « après tout ça vaut peut-être la peine d'aller faire deux heures d'histoire et de géographie».

75 - AS. D'où les thèmes nouveaux comme l'énergie. Le thème $4 \mathrm{du}$ programme mêle à la fois un type d'espace, les littoraux, qui est assez clairement identifié et traditionnel, qui était obligatoire dans le programme précédent, puis un thème reformulé, les espaces soumis aux risques majeurs, qui renvoie donc à une problématique et une grille de lecture actuelle qui est le risque. Enfin, un thème totalement nouveau, l'Arctique qui est une région du monde. Qu'est-ce qui a présidé à l'organisation de ce thème 4 ?

76 - FL. La logique, c'est une logique par type d'entrées. A la suite des entrées thématiques transversales (alimentation, énergie, ...), on a des types d'espaces. Il y a une façon d'essayer de ne pas être en retard sur les problématiques actuelles. L'idée, c'est d'aborder des types d'espaces habituels et en même temps de la nouveauté... Alors: pourquoi l'Arctique ? C'est un très, très beau thème. Longtemps on a hésité avec la mer ... On est obligé de faire des choix. Je pense que traiter l'Arctique dans un programme qui a comme fil directeur le développement durable, n'est pas absurde du tout... Certes c'est un espace très particulier, mais aussi très emblématique, ce qui en fait un espace attractif voire attachant pour l'étude en classe. Cela pèse dans les choix aussi.

77 - AS. La dimension aventure de la géographie en somme...

78 - FL. Les programmes, c'est cela aussi. Nous avons des objectifs pédagogiques généraux avec une formation intellectuelle, culturelle et civique, en sachant qu'il faut éviter l'accumulation, qu'il faut faire des choix. L'idée qu'il faut acquérir un certain nombre de techniques et de réflexes intellectuels à travers des exercices et des cas variés. Enfin, il y a aussi l'idée qu'il faut essayer de rendre les programmes attractifs pour intéresser des jeunes. D'où l'idée ponctuellement d'avoir aussi des thèmes accrocheurs en lien avec des recherches récentes comme l'Arctique.

79 - AS. La géographie, c'est aussi voyager et rêver...

80 - FL. Oui, c'est largement le programme de 6e qui a été complètement revu autour de cela mais c'est un autre sujet.

81 - AS. Sur cette invitation au rêve et au voyage, François Louveaux, merci !

\section{AUTEUR}

\section{ALEXIS SIERRA}

Alexis Sierra est maître de conférences en géographie à l'université Cergy-Pontoise-IUFM.

alexisierra2001@yahoo.fr 